\title{
TECHNIQUES AND APPLICATIONS
}

\section{RNAi 'off-targets' pathogen infection}

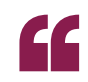

\section{in addition \\ to on-target} mRNA-specific effects, seedspecific offtarget effects are important determinants of pathogen infection

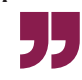

RNA interference (RNAi) has been used to identify host factors that influence infection of human cells with bacterial and viral pathogens. However, little overlap was seen in the results of different screens, and many of the identified factors were subsequently shown to be false positives in follow-up studies, presumably owing to off-target effects of the small interfering RNAs (siRNAs) that were used for RNAi. Franceschini et al. now show that these off-target effects are specific and depend on the sequence of nucleotides 2-8 (also known as the seed sequence) of the siRNA.

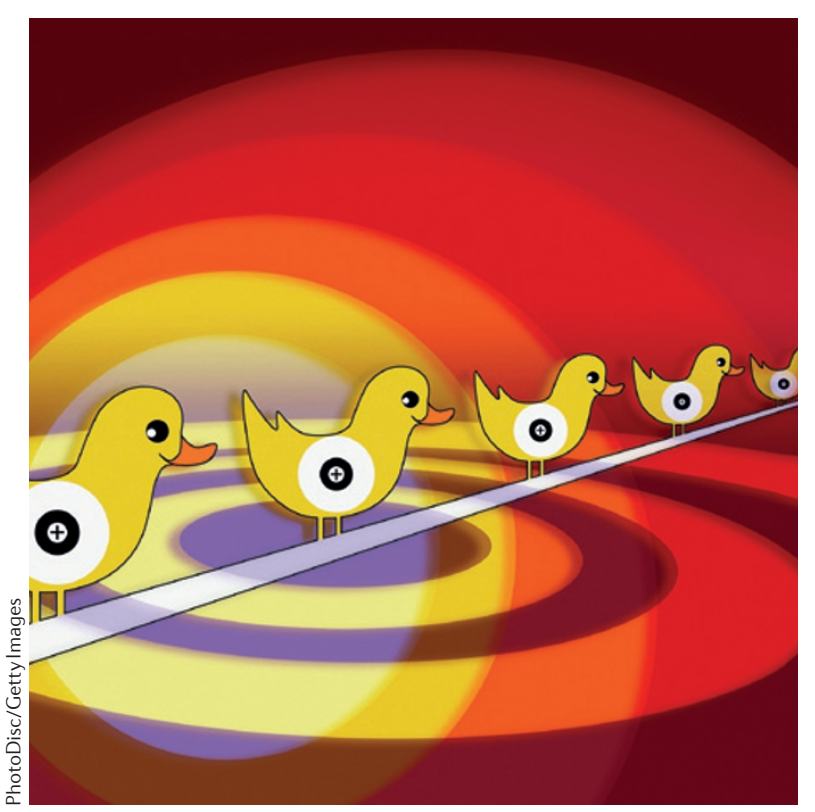

RNAi functions via posttranscriptional silencing of mRNAs, which can be targeted by siRNAs that are fully complementary to the mRNA sequence or by microRNAs (miRNAs) that show a partial sequence match. Libraries of siRNAs that target human mRNAs can be used to screen the role of host factors during pathogen infection. When Franceschini et al. compared several siRNA libraries in such a screen, they noticed almost no correlation in the ability of siRNAs from different vendors targeting the same mRNA to influence the infection of cells with Brucella abortus, Salmonella enterica subsp. enterica serovar Typhimurium and Uukuniemi virus, as measured by automated fluorescence microscopy. However, when the authors analysed the results on the basis of matching the seed sequences instead of the target mRNA sequences, they found strong correlations. One-third of all tested siRNAs reproducibly inhibited or promoted pathogen infection, and siRNAs that had matching seed sequences showed the same activity despite having different mRNA targets, which suggests that the seed sequences mediate specific off-target effects.

To test this hypothesis, the authors designed synthetic siRNAs that contained inhibiting, neutral or promoting seed sequences, as identified in the previous library screen, followed by a random sequence that was not complementary to any human mRNA. These synthetic siRNAs indeed showed the infection phenotype that was predicted by their seed sequence. Furthermore, when point mutations were introduced into the seed sequence, the effects on infection were abolished, which indicates that the off-target effects are sequence specific. The authors thus suggest that the active siRNAs might function as miRNAs owing to partial sequence matches with off-target mRNAs via their seeds. However, given the notorious difficulties of computationally predicting miRNA targets, they were unable to derive further mechanistic explanations using databases of seed-sequence matches.

Nevertheless, this study shows that the results of siRNA screens need to be carefully evaluated and that, in addition to on-target mRNA-specific effects, seed-specific off-target effects are important determinants of pathogen infection. Furthermore, the authors suggest that, based on this information, novel RNAs could be designed that reproducibly block infection, which they term 'seed drugs.

Ursula Hofer

\section{ORIGINAL RESEARCH PAPER}

Franceschini, A. et al. Specific inhibition of diverse pathogens in human cells by synthetic microRNAlike oligonucleotides inferred from RNAi screens.

Proc. Natl Acad. Sci. USA http://dx.doi. org/10.1073/pnas.1402353111 (2014) 